Sādhanā Vol. 40, Part 1, February 2015, pp. 107-119. (C) Indian Academy of Sciences

\title{
Fuzzy delay model based fault simulator for crosstalk delay fault test generation in asynchronous sequential circuits
}

\author{
S JAYANTHY $^{1, *}$ and M C BHUVANESWARI ${ }^{2}$ \\ ${ }^{1}$ Department of Electronics and Communication Engineering (ECE), Sri \\ Ramakrishna Engineering College, Coimbatore 641 022, India \\ ${ }^{2}$ Department of Electrical and Electronics Engineering (EEE), P.S.G. College of \\ Technology, Coimbatore 641 004, India \\ e-mail: jayanthy.s@srec.ac.in; mcb@eee.psgtech.ac.in
}

MS received 23 February 2014; accepted 11 September 2014

\begin{abstract}
In this paper, a fuzzy delay model based crosstalk delay fault simulator is proposed. As design trends move towards nanometer technologies, more number of new parameters affects the delay of the component. Fuzzy delay models are ideal for modelling the uncertainty found in the design and manufacturing steps. The fault simulator based on fuzzy delay detects unstable states, oscillations and non-confluence of settling states in asynchronous sequential circuits. The fuzzy delay model based fault simulator is used to validate the test patterns produced by Elitist Non-dominated sorting Genetic Algorithm (ENGA) based test generator, for detecting crosstalk delay faults in asynchronous sequential circuits. The multi-objective genetic algorithm, ENGA targets two objectives of maximizing fault coverage and minimizing number of transitions. Experimental results are tabulated for SIS benchmark circuits for three gate delay models, namely unit delay model, rise/fall delay model and fuzzy delay model. Experimental results indicate that test validation using fuzzy delay model is more accurate than unit delay model and rise/fall delay model.
\end{abstract}

Keywords. Asynchronous sequential circuits; crosstalk delay faults; fuzzy delay model; multi-objective genetic algorithm; fault coverage; transitions.

\section{Introduction}

For deep submicron designs with frequency in the gigahertz range and high circuit density, asynchronous systems are viewed as an alternative to purely synchronous systems due to clock skew, power dissipation and noise issues (Al-Assadi \& Kakarla 2009). Asynchronous circuits have high performance gains and low power when compared to their synchronous counterparts. The types of error to be tested in asynchronous circuits are stuck at fault errors and delay faults. The latter can disrupt proper operation of the circuit. The problem with asynchronous test

*For correspondence 
generation is that the test patterns must guarantee not only that the deterministic fault effect is captured correctly, but also that the fault effect is not overwritten and does not disappear during the subsequent operations. This complicates the fault simulation and automatic test pattern generation processes significantly.

In asynchronous circuits, elaborate timing relationships must be verified and tested for any manufacturing defects. The asynchronous circuits, cycle through several unstable states before settling in a stable state. The final state of the system may be different depending on the arrival time of the input events and the delays of the internal gates and interconnection. Hence an accurate modelling of the delay is necessary. During simulation of the asynchronous circuits the hazards, unstable states, oscillations and non-confluence of settling states must be taken into consideration.

In digital circuits, the delays associated with each element are different and may not be known with precision for a given fabrication technology. The delays of a logic gates or interconnect are affected by various fabrication process parameters and however accurate the delay models are, it is very difficult to model the process uncertainties.

In this paper, the fuzzy delay model is employed for test generation of crosstalk delay faults in asynchronous sequential circuits. The fuzzy delay model can handle uncertainties with respect to timing characteristics and manufacturing anomalies. It can be used to detect oscillations and non-confluence of settling states, caused due to delays in the input transitions and gates.

With the intensive scaling in process technology and increase in switching speed, the problem of modelling gate delays becomes more difficult. In deep sub micron VLSI designs, the portion of delay contributed by gates reduces while interconnect delay becomes dominant. Of all the various delay effects, the cross coupling capacitance or crosstalk noise effects are considered to have a predominant in digital circuits that may result in improper functioning of a chip (Aragones et al 2002). There are two main types of crosstalk effects: crosstalk glitch and crosstalk-induced delay. A crosstalk glitch is a pulse that is induced by coupling effects among interconnects lines. Crosstalk delay is a signal delay induced by the same coupling effects, that is simultaneous transitions among interconnect lines. Crosstalk causes a delay in addition to normal gate delay and interconnects delay.

In this paper, the fuzzy delay model based fault simulator is used, for test generation of crosstalk delay faults in asynchronous sequential circuits. To optimize the fault coverage, multiobjective genetic algorithm technique, ENGA (Deb 2002), which targets two objectives of maximizing fault coverage and minimizing number of transitions is employed. Minimizing the number of transitions reduces the power dissipation in a circuit.

Section 2 reviews the previous works on test generation and simulation of asynchronous sequential circuits. Fuzzy delay model is discussed in section 3. Event driven simulation algorithm based on fuzzy delay model is described in section 4. Section 5 gives a brief description of crosstalk delay fault simulator with fuzzy delay for gates. Section 6 presents the experimental results for asynchronous SIS benchmark circuits. Conclusions are provided in section 7.

\section{Related work on test generation and simulation}

Delay testing of asynchronous circuits can be reduced to a problem of test pattern generation for a combinational circuit as shown in Kishinevsky et al (1998). The asynchronous circuit is converted into an asynchronous net with local feedback loops, simple gates and latches. A set of equations is formulated to find paths whose delay was within the permissible limits. The 
asynchronous net was converted into an iterative combinational array with initial values and a robust path delay fault testing was used to generate test vectors for path delay faults.

A serial fault simulator for testing gate delay faults and transition faults in asynchronous circuits was designed by Sur-Kolay et al (2000). Min-max timing analysis was used to compute the signal delays. The asynchronous circuit was unfolded into timeframes and simulated. The time stamps for the primary output signals at the end of each time frame are checked to see if the delay was lower than the corresponding delay for the fault-free simulation.

Shi and Makris (2006) used a design for test technique for testing delay faults in asynchronous handshake circuits. Delay faults were tested using a multiplexer based full scan method.

Michele Favalli (2005) has proposed fuzzy model for testing path delay faults. Non- robust path delay fault model is used to detect delay faults. To find the quality of non-robust tests, a fuzzy delay model was used. Monte-Carlo simulation was used to evaluate test pairs which detect delay faults and this data was compared with the ranking obtained from fuzzy model implemented in a path delay fault simulator. The results obtained for combinational circuits show that fuzzy delay for testing path delay faults was more accurate.

The above papers dealt with gate delay and path delay faults. In this paper, a fuzzy delay model is used to model the gate delay for testing crosstalk delay faults. The fuzzy delay model is implemented in a crosstalk delay fault simulator. Multi-objective Genetic Algorithm, which is a simulation based test generation algorithm, is used for generating optimized test patterns for testing crosstalk delay faults in asynchronous sequential circuits. The test patterns are validated using the fuzzy delay based crosstalk delay fault simulator.

\section{Fuzzy delay model}

The delays of a logic gate or interconnect is effected by various fabrication process parameters and however accurate the delay models are it is difficult to model the process uncertainties. The fuzzy delay model can handle uncertainties with respect to timing characteristics and manufacturing anomalies. In fuzzy delay model, the delay is represented by a possibility distribution. The possibility distribution of gate delay is a statistical function that describes all the possible values and likelihoods that a gate delay can take within a given range.

In fuzzy delay model (Giambiasi et al 1994; Jayabharathi et al 1999), as shown in figure 1, the delay $F_{d}$ is represented by a possibility distribution $\pi_{\mathrm{fdm}}$. In order to have a model which

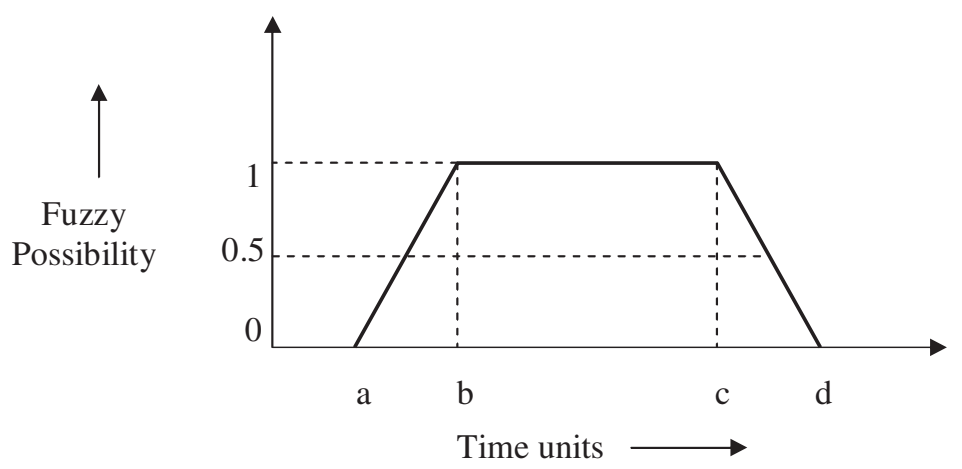

Figure 1. Fuzzy delay model. 
gives reliable modeling of the imperfect information's concerning the delays, $\pi_{\mathrm{fdm}}$ is defined by Eq. (1).

$$
\left.\pi_{\mathrm{fdm}}(\mathrm{t})=\begin{array}{ll}
0 & \text { if }(\mathrm{t} \leq \mathrm{a}) \text { or }(\mathrm{t} \geq \mathrm{d}) \\
(\mathrm{t}-\mathrm{a}) /(\mathrm{b}-\mathrm{a}) & \text { if }(\mathrm{a} \leq \mathrm{t} \leq \mathrm{b}) \\
1 & \text { if }(\mathrm{b} \leq \mathrm{t} \leq \mathrm{c}) \\
(\mathrm{t}-\mathrm{d}) /(\mathrm{c}-\mathrm{d}) & \text { if }(\mathrm{c} \leq \mathrm{t} \leq \mathrm{d})
\end{array}\right\} \text {, }
$$

where the fuzzy dates $\mathrm{a}, \mathrm{b}, \mathrm{c}$ and $\mathrm{d}$ are elements of the reference set $\mathrm{T}$ (time scale). The fuzzy delay $F_{d}$ is defined by the quadruplet $(a, b, c, d)$.

The range of values between $a$ and $b$, or $c$ and $d$ in the fuzzy delay model in figure 1 has a grade of membership function. Thus, by using possibility distribution, the fuzzy delay model provides an environment for a more accurate representation of uncertainty and vague concepts as compared to the other types of delay models. Every manufacturing process is characterized using a test chip and the data collected can be used for developing timing models. The manufacturing defect data can be collected and used to create defect densities and to analyse layout sensitivities. A fuzzy rule base can be created using the defect data and the process timing models.

\subsection{Representation of fuzzy delay}

The union and intersection operator can be used to propagate the fuzzy delays along a path. For example, consider the OR gate with two inputs $i_{1}$ and $i_{2}$ and one output C. Let $\pi_{i 1}$ and $\pi_{i 2}$ the fuzzy delays of the 0 to 1 transitions of the input $i_{1}$ and $i_{2}$, respectively. The output 0 to 1 transition depends on the earliest 0 to 1 transition on its inputs. The fuzzy delay of the output transition can be represented using the fuzzy arithmetic operators max and min as given in Eq. (2).

$$
\pi_{c 0 \rightarrow 1}=\max \left(\min \left(\pi_{i 1}, \pi_{i 2}(-\infty, t)\right), \min \left(\pi_{i 2}, \pi_{i 1}(-\infty, t)\right)\right),
$$

where $\pi_{i 1}(-\infty, t)$ and $\pi_{i 2}(-\infty, t)$ represent the fuzzy set of time points before the respective transitions. That is $\pi_{i 1}(-\infty, t)$ represent the condition when the input $i_{1}$, is stable at zero. Equation (2) can be generalized to include different gate types and transitions (Kandel 1986; Dubois \& Prade 1989; Giambiasi et al 1994).

\section{Discrete event driven logic simulation based on fuzzy delay model}

This section presents a gate level logic simulation algorithm for asynchronous sequential circuits. The delay model used is the fuzzy delay model. The fundamental mode of operation is assumed, where signals at the primary inputs are allowed to change only after the signals at the primary outputs have been stabilized. It is assumed that the gate delay bounds are known in advance for a given circuit and process technology. The component delays for both rise and fall transitions are represented as fuzzy delays. The effect of the fanouts and the input slope are considered in generating the delays. A discrete event simulation model assumes that the system being simulated changes only at discrete points in simulated time. Each event contains a time stamp and denotes a change in the state of the system. The simulator typically removes the smallest time-stamped event from the event list and processes that event. Processing an event involves executing some simulator code to effect the appropriate change in state and scheduling one or more events. The simulation technique can be applied to models other than logic gates (Bhuvaneswari \& Sivanandam 2000). 
The discrete event simulation of the fuzzy delay model involves two difficulties:

- The logical state changes occur at fuzzy dates covering time intervals that overlap.

- The conceptual model is continuous; a discrete model equivalent has to be defined (Banks et al 1996).

To overcome these two difficulties, a discrete event driven logic simulation model based on the concepts of states and events is used (Giambiasi et al 1994).

The state of a component $\mathrm{D}$ is described by two variables: state-at-logic 0 (state- $0_{\mathrm{d}}$ ) and stateat-logic 1 (state- $\left.1_{\mathrm{d}}\right)$. The two variables are defined by the triplets $\left(t_{d}^{0}, \pi_{d}^{0}, \partial_{d}^{0}\right)$ and $\left(t_{d}^{1}, \pi_{d}^{1}, \partial_{d}^{1},\right)$ where for every $\mathrm{S}$ belonging to $\{0,1\}$ :

$t_{d}^{s}$ - is the date at which last change of the variable state-at- $S_{d}$ occurs.

$\pi_{d}^{s}$ - is the degree of possibility that d may be in the state $\mathrm{S}$ at the date $t_{d}^{s}$.

$\partial_{d}^{s}$ - is the slope that orients the function describing the possibility of $\mathrm{d}$ to be in the state $\mathrm{S}$ beginning at the date $t_{d}^{s}$.

A state change in the discrete event simulation model is defined as a discrete change of at least one of the slopes that orient the function describing the possibility of a component to be in the state at logic 0 or 1 . An event is associated with this state change. The date of occurrence of the event is the date at which the slope change occurs.

An event is defined by quintuplet $(\mathrm{t}, \mathrm{p}, \delta, \mathrm{S}, \mathrm{D})$, where $(\mathrm{t}, \mathrm{p}, \delta)$ represent the state at $\mathrm{S}$ of the component $\mathrm{D}$ in which the fuzzy event has occurred at fuzzy date $\mathrm{t}$.

For example, consider the input $i_{1}$ of a logic gate changing from logic state 0 to 1 at fuzzy date $F_{d}(1,2,2,3)$. The initial state-at- 0 of $i_{1}$ is $(0,1,0)$ as illustrated in figure 2 . Two events $(2$, $\left.1,-1,0, i_{1}\right)$ and $\left(3,0,0,0, i_{1}\right)$ are created at the fuzzy dates 2 and 3 , respectively for the state-at- 0 , as illustrated in figure 2 .

Similarly, two events $\left(1,0,1,1, i_{1}\right)$ and $\left(2,1,0,1, i_{1}\right)$ are created at fuzzy dates 1 and 2 , respectively for the variable state-at-1. Thus, four events are created for a input transition with fuzzy delay $F_{d}$.

An event $(\mathrm{t}, \mathrm{p}, \delta, \mathrm{e}, \mathrm{d})$ is compatible with state $\left(t_{\alpha}^{0}, \pi_{\alpha}^{0}, \partial_{\alpha}^{0}\right),\left(t_{\alpha}^{1}, \pi_{\alpha}^{1}, \partial_{\alpha}^{1}\right)$ of a component $\alpha$ when $\mathrm{c}=\alpha$, as shown in Eq. (3).

$$
\left.\begin{array}{l}
\delta \neq \delta_{\alpha}^{0} \text { et } \partial=\frac{\pi_{\alpha}^{0}-\pi}{t_{\alpha}^{0}-t}, \text { if } \mathrm{e}=0 \\
\delta \neq \delta_{\alpha}^{1} \text { et } \partial=\frac{\pi_{\alpha}^{1}-\pi}{t_{\alpha}^{1}-t}, \text { if } \mathrm{e}=1
\end{array}\right\} .
$$

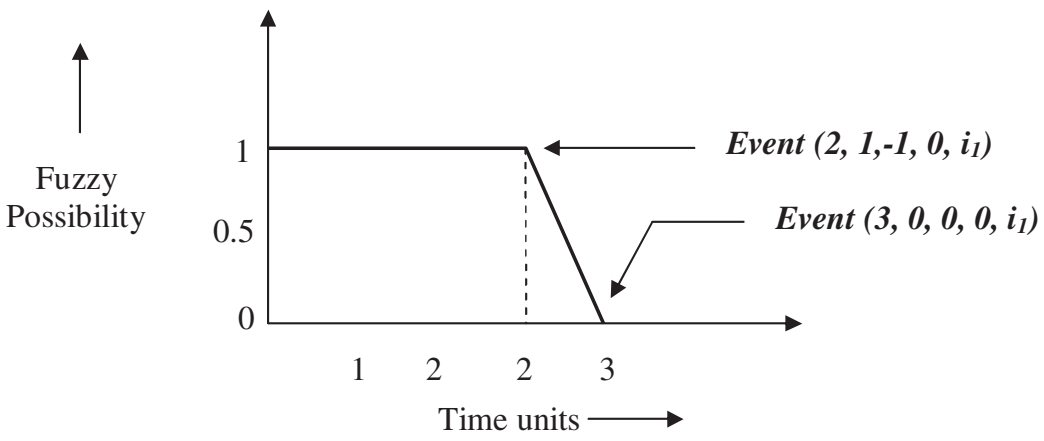

Figure 2. Events created at state at 0 of $i_{1}$. 


\subsection{Processing of events}

The processing of the events depends on the type of the component $\mathrm{D}$. The component may be a primary input, input of the logic element, output of the delay element or the primary output (Giambiasi et al 1994). The processing of the event $\mathrm{E}$ ( $\mathrm{t}, \mathrm{p}, \delta, \mathrm{S}, \mathrm{D})$ on $\mathrm{D}$ is detailed below.

(i) $\mathrm{D}$ is a primary input: The event $\mathrm{E}$ on $\mathrm{D}$ may provoke a state change on each successor $\alpha$. If $\alpha$ is an output of a logic element of type positive gate (negative gate), the possible state change on $\alpha$ is expressed by the event ( $\mathrm{t}, \mathrm{p}, \delta, \mathrm{S}, \alpha)(\mathrm{t}, \mathrm{p}, \delta, \mathrm{S} / \alpha)$, where $\mathrm{S}$ / the complement of state-at-S.

(ii) $\mathrm{D}$ is the output of any logic element: If the event $\mathrm{E}$ is compatible with state-at-S of the component $\mathrm{D}$ with fuzzy delay $\pi_{f d}$, the event may provoke a state change on each successor $\alpha$ (ie) output of the delay element, expressed by the event $\left(\mathrm{t}+t^{\prime}, \mathrm{p}\right.$, combine $\left.\left(\delta, \delta^{\prime}\right), \mathrm{S}, \alpha\right)$ where $\delta^{\prime}$ upslope of the $\pi_{f d}$ if $\delta>0$ and the down slope of $\pi_{f d}$ if $\delta<0, t^{\prime}$ is the time point that corresponds to the possibility $\pi$ of $\delta$, shown in Eq. (4).

$$
\text { Combine } \left.\quad \begin{array}{rl}
\left(\delta, \delta^{\prime}\right) & =\frac{\delta * \delta^{\prime}}{\delta+\delta^{\prime}} \quad \text { if } \delta \neq \pm \infty \text { and } \delta^{\prime} \neq \pm \infty \\
& =\delta \text { if } \delta \neq \pm \infty \text { and } \delta^{\prime} \neq \pm \infty \\
& =\delta^{\prime} \text { otherwise }
\end{array}\right\} .
$$

If the event $\mathrm{E}$ is not compatible with state-at-S of $\alpha$, an $E^{\prime}\left(t^{\prime}, p^{\prime}, \delta, S, D\right)$ is produced on the component $\mathrm{D}$, if $(\mathrm{t}, \mathrm{p}, \delta)$ intersects with state-at-S of $\mathrm{D}$, where $\left(t^{\prime}, p^{\prime}\right)$ is the intersection point.

(iii) $\mathrm{D}$ is the output of a delay element: If the event $\mathrm{E}$ is compatible with the state at $\mathrm{S}$ of $\mathrm{D}$, then the event ( $\mathrm{t}, \pi, a, S, \alpha)$ or $\left(\mathrm{t}, \pi, a, S^{\prime}, \alpha\right)$ is created based on the type of the gate. If the event $\mathrm{E}$ is not compatible with the state at $\mathrm{S}$ of $\mathrm{D}$, then an event $E^{\prime}$ is created on itself.

(iv) $\mathrm{D}$ is a primary output. If the event $\mathrm{E}$ is compatible with state-at-S of the component $\mathrm{D}$, the value of state-at-S changes to $(\mathrm{t}, \mathrm{p}, \delta)$.

\section{Crosstalk delay fault simulation algorithm}

Given a test vector sequence and target fault list as inputs, the objective of the fuzzy delay model based crosstalk delay fault simulator is to determine the number of faults detected by the test sequence generated by the ENGA based ATPG. The target fault list consists of crosstalk delay faults derived by static timing analysis (Takahashi et al 2005). Using both the topological and timing information, static timing analysis generates a set of target crosstalk delay faults. The asynchronous sequential circuit is converted into timeframe of combinational circuit with Pseudo Primary Inputs (PPI) and Pseudo Primary Outputs (PPO) using a feedback path identification algorithm (Sur-Kolay et al 2000). Static timing analysis is performed to the converted asynchronous circuits with PPI and PPO. The fault simulator uses event driven technique as described in section 4 . The fault simulator is capable of detecting single victim/multiple aggressor crosstalk delay faults. The fault is detected if a delay effect is observed at the PO. The algorithm for crosstalk delay fault simulation consists of the following steps:

\section{Step 1: Initialization of the circuit}

This step allows the circuit to be initialized for the first test vector and the state of all the components of the circuit is initialized. 


\section{Step 2: Creation of the initial events and initialization of the scheduler}

The aim of this step is to create the corresponding events as described in section 4 for the second test vector. The events are inserted into the event list.

\section{Step 3: Development of the fault simulation}

While the schedule is not empty the following are repeated.

(i) Current time takes its value the occurrence time of the event that is at the top of the schedule.

(ii) The events having an occurrence time equal to the current time is inserted in the current events.

(iii) While current events are not empty do

(a) Extract the first event of current time and process it according to the element concerned. The processing of events is as described in section 4.1.

(b) If the events have been created, then insert those having an occurrence time equal to value of current time in the current events and the others in the schedule.

(c) Crosstalk delay faults are read from the fault list. All aggressors for a particular victim are read and simultaneously checked for activation. Faults are activated when victim and aggressors have opposite transition. If the fault is activated, the fault is injected by inserting the victim events in the schedule at a time instant equal to the estimated delay. The delay is calculated by coupling capacitance and resistance value from the cell library. Other events are scheduled for a good circuit as in step 3(b).

(d) The events are processed and if the delay effect is observed at the primary output event, the fault is detected and removed from the fault list.

Detection of oscillation: The simulation of the circuit that oscillates will result in repeated scheduling and processing of the same sequence of events, with the simulation program apparently caught in an endless loop. The detection of oscillation is performed by counting the number of events generated. When this number exceeds a predefined value (50), the simulator assumes that an oscillation has occurred.

Detection of non-confluence of settling state: When simulation completes with incompatible probabilities for state-at- 0 and state-at-1, it was assumed that non-confluence of settling states has occurred. For example, non-confluence is said to have occurred, if the state- at- 1 and state-at-0 variables of the primary output have the same probabilities i.e, $(\mathrm{t}, 0,0)$ and $\left(\mathrm{t}^{\prime}, 0,0\right)$, respectively (Bhuvaneswari 2001).

\section{Experimental results}

The serial fault simulator for crosstalk delay faults using fuzzy delay model for gates was implemented in INTEL core I7 processor with $2.6 \mathrm{GHz}$ and 4GB RAM using C language in Linux environment.

The delay for each element was derived using a $45 \mathrm{~nm}$ Nangate generic open cell library (Benchmark site 1). Fuzzy delay model for rising and falling transitions of the logic gates and zero wire delays are assumed. The activated victim events are given a delay calculated by using coupling capacitance and resistance value from cell library. 
Table 1. The SIS Benchmark circuit characteristics.

\begin{tabular}{lccccccc}
\hline Circuit & $\begin{array}{c}\text { No. of } \\
\text { PI }\end{array}$ & $\begin{array}{c}\text { No. of } \\
\text { PO }\end{array}$ & $\begin{array}{c}\text { No. of Gates } \\
\text { +Latches }\end{array}$ & $\begin{array}{c}\text { No. of feedback } \\
\text { paths }\end{array}$ & $\begin{array}{c}\text { Total No. } \\
\text { of paths }\end{array}$ & $\begin{array}{c}\text { No. of Critical } \\
\text { paths }\end{array}$ & $\begin{array}{r}\text { Total no. } \\
\text { of faults }\end{array}$ \\
\hline alloc_outbound & 4 & 5 & 15 & 2 & 16 & 3 & 197 \\
chu172 & 3 & 3 & 9 & 0 & 7 & 1 & 34 \\
dff & 2 & 1 & 9 & 2 & 8 & 3 & 60 \\
isend & 4 & 6 & 42 & 6 & 66 & 1 & 390 \\
nak-pa & 4 & 6 & 20 & 1 & 20 & 1 & 75 \\
sbuf-send-ct1 & 3 & 5 & 19 & 3 & 18 & 1 & 177 \\
sbuf-send-pkt2 & 4 & 5 & 18 & 2 & 24 & 1 & 150 \\
ram-read & 5 & 6 & 23 & 3 & 23 & 1 & 109 \\
\hline
\end{tabular}

Table 1 gives the characteristics of SIS benchmark circuits synthesized using Petrify (Benchmark site 2). The circuit name, the number of Primary Inputs (PI), number of Primary Outputs (PO), number of gates, number of feedback paths, number of paths, number of critical paths and the reduced set of target crosstalk faults derived by Static Timing Analysis corresponding to each circuit are given.

Initially, random test patterns (50) are generated using a pseudo random process for SIS benchmark circuits. The random test patterns are validated using fuzzy delay model based fault simulator. The results for random patterns are tabulated in table 2 . The fault coverage and number of transitions are given for the benchmark circuits. The numbers of test vectors that are invalidated by the fuzzy delay model are also shown in the table 2 . The test vectors that cause oscillations and non-confluence of settling states are invalidated by the fuzzy delay based fault simulator.

To increase the fault coverage multi-objective genetic algorithms WSGA, ENGA and ENGA with redundancy (Jayanthy et al 2013) are applied to several SIS benchmark circuits. The test vectors generated by WSGA based ATPG, ENGA based ATPG and ENGA based ATPG with redundancy are validated using fuzzy delay based fault simulator. The number of generations for the genetic algorithms based ATPGs is assumed to be 2 or 3 for smaller benchmark circuits and 4 or 5 for larger benchmark circuits. A population size in the range of 2 to 4 is used. A crossover probability of 1 and mutation probability of 0.1 are used. The selection scheme used is the binary tournament selection. The redundancy factor of 5 is chosen. By experimental results it is observed that this redundancy factor gives better fault coverage with less number of transitions for all the benchmark circuits.

Table 2. Experimental results for random patterns.

\begin{tabular}{lccc}
\hline Circuit & Fault coverage (\%) & No. of transitions & Tests invalidated (\%) \\
\hline alloc_outbound & 44.16 & 353 & 4 \\
chu172 & 58.82 & 48 & 0 \\
dff & 40 & 164 & 28 \\
isend & 34.62 & 276 & 12 \\
nak-pa & 33.33 & 355 & 6 \\
sbuf-send-ct1 & 13.55 & 121 & 20 \\
sbuf-send-pkt2 & 18.66 & 105 & 16 \\
ram-read & 37.61 & 121 & 12 \\
\hline
\end{tabular}


Experiments results illustrated in table 3 are performed using the WSGA based test generator. Results for the four crossover operator's namely; uniform crossover, one-point crossover, two-point crossover and weight based crossover are tabulated. The bold number represents minimum number of transitions for each benchmark circuit. Of the four crossover schemes, uniform crossover gives less number of transitions for 4 out of 8 benchmark circuits. The fault coverage and CPU time are nearly equal for all the crossover schemes since the same progress limit for test generation is used. Compared to random vectors WSGA based ATPG gives a better fault coverage and reduced number of transitions.

Experimental results using ENGA based ATPG are given in table 4. The number of faults detected and number of transitions for the various crossover schemes is compared. The bold

Table 3. Test results for WSGA based ATPG.

\begin{tabular}{lccccc}
\hline \multirow{2}{*}{ Circuit } & No. of faults detected & \multicolumn{4}{c}{ No. of transitions } \\
\cline { 3 - 5 } alloc_outbound & 122 & $\mathbf{3 1 4}$ & 315 & 324 & 324 \\
chu172 & 26 & 200 & 229 & 203 & $\mathbf{1 9 5}$ \\
dff & 41 & 326 & $\mathbf{3 0 7}$ & 308 & 333 \\
isend & 228 & 680 & $\mathbf{6 1 3}$ & 630 & 664 \\
nak-pa & 48 & $\mathbf{5 4 4}$ & 611 & 634 & 640 \\
sbuf-send-ct1 & 115 & $\mathbf{5 8 5}$ & 626 & 639 & 629 \\
sbuf-send-pkt2 & 102 & 583 & 584 & $\mathbf{5 3 7}$ & 556 \\
ram-read & 60 & $\mathbf{4 5 8}$ & 502 & 520 & 524 \\
\hline
\end{tabular}

Table 4. Test results for ENGA based ATPG.

\begin{tabular}{lccccc}
\hline \multirow{2}{*}{ Circuit } & No. of faults detected & \multicolumn{4}{c}{ No. of transitions } \\
\cline { 3 - 5 } alloc_outbound & 121 & $\mathbf{2 0 8}$ & 210 & 211 & 211 \\
chu172 & 27 & 199 & 202 & 195 & $\mathbf{1 9 4}$ \\
dff & 36 & $\mathbf{1 0 2}$ & 112 & 114 & 119 \\
isend & 216 & $\mathbf{5 1 7}$ & 518 & 524 & 526 \\
nak-pa & 55 & 439 & $\mathbf{4 2 2}$ & 441 & 431 \\
sbuf-send-ct1 & 117 & 296 & $\mathbf{2 9 3}$ & 294 & 298 \\
sbuf-send-pkt2 & 109 & $\mathbf{5 0 7}$ & 526 & 529 & 519 \\
ram-read & 64 & $\mathbf{2 7 6}$ & 278 & 314 & 314 \\
\hline
\end{tabular}

Table 5. Test results for ENGA based ATPG with redundancy.

\begin{tabular}{lcrrrrrr}
\hline \multirow{2}{*}{ Circuit } & $\begin{array}{c}\text { No. of faults } \\
\text { detected }\end{array}$ & \multicolumn{4}{c}{ No. of transitions } & $\begin{array}{c}\text { No. of test } \\
\text { vectors }\end{array}$ & CPU time(sec) \\
\cline { 3 - 6 } alloc_outbound & 115 & $\mathbf{1 0 2}$ & 104 & 105 & 105 & 16 & 0.27 \\
chu172 & 27 & 91 & 94 & $\mathbf{8 7}$ & 89 & 32 & 0.27 \\
dff & 32 & $\mathbf{5 8}$ & 59 & 60 & 61 & 8 & 0.19 \\
isend & 216 & 253 & $\mathbf{2 5 2}$ & 260 & 258 & 16 & 0.56 \\
nak-pa & 45 & $\mathbf{1 6 7}$ & 168 & 170 & 168 & 32 & 0.36 \\
sbuf-send-ct1 & 112 & 126 & $\mathbf{1 2 3}$ & 131 & 128 & 32 & 0.23 \\
sbuf-send-pkt2 & 108 & $\mathbf{2 2 3}$ & 242 & 264 & 224 & 32 & 0.38 \\
ram-read & 62 & $\mathbf{2 1 7}$ & 221 & 229 & 230 & 24 & 0.27 \\
\hline
\end{tabular}


number represents minimum number of transitions for each circuit. Of the four crossover schemes, uniform crossover gives less number of transitions for 5 out of 8 benchmark circuits.

The experimental results for ENGA based ATPG with redundancy are shown in table 5 for the various crossover schemes. The bold number represents minimum number of transitions for each circuit. Out of the four crossover schemes, uniform crossover gives less number of transitions for 5 out of 8 benchmark circuits. The fault coverage and number of test vectors are nearly equal for all the crossover operators. The CPU time in seconds gives the execution time of the ENGA based ATPG with redundancy. The CPU time is nearly equal for all the test generators.

Table 6 gives a comparison of fault coverage and number of transitions for WSGA based ATPG, ENGA based ATPG and ENGA based ATPG with redundancy using fuzzy delay based crosstalk delay fault simulator. ENGA based ATPG with redundancy gives a better fault coverage and reduced number of transitions compared with the other ATPGs. The bold number represents the maximum number of faults detected and minimum number of transitions obtained for each circuit. The results are taken with uniform crossover. It is observed that with a slight increase in the fault coverage of $\mathbf{1 . 2 3} \%$ ENGA based ATPG achieves a reduction in transition activity of $\mathbf{3 1 . 0 1} \%$. When redundancy is introduced in the ENGA based ATPG the fault coverage is decreased by $\mathbf{2 . 3 2 \%}$ but the method achieved a good reduction in number of transitions of about $66.15 \%$.

Table 7 summarizes the results for ENGA based ATPG with redundancy using unit delay simulator, rise/fall delay simulator and fuzzy delay simulator. Results are obtained using uniform crossover. In the unit delay crosstalk delay fault simulator for asynchronous sequential circuits gates were assumed unit delay and delay injected is also one unit. In the rise/fall delay crosstalk delay fault simulator for asynchronous sequential circuits gates were assigned a rise/fall delay and delay to be injected is calculated by coupling capacitance and resistance value (Jayanthy et al 2013).

The number of faults detected and number of transitions are compared. Compared to ENGA based ATPG using unit delay simulator, the fault coverage decreases for rise/fall delay fault simulator and hence number of transitions is also less. This is because timing is more accurate in rise/fall delay simulator and hence fault effect at the primary output is also more accurate. This model also considers the effect of input signal slope, in addition to the loading characteristics. CPU time is higher for ENGA based ATPG using rise/fall delay fault simulator as delays are calculated from the cell library. Compared to the other two test generators, ENGA based ATPG using fuzzy delay simulator gives comparatively lower fault coverage. This is because the tests that cause oscillations and non-confluence of settling states are identified and invalidated by the

Table 7. Test results using ENGA based ATPG with redundancy.

\begin{tabular}{|c|c|c|c|c|c|c|}
\hline \multirow[b]{2}{*}{ Circuit } & \multicolumn{2}{|c|}{ Unit delay } & \multicolumn{2}{|c|}{ Rise/Fall delay } & \multicolumn{2}{|c|}{ Fuzzy delay } \\
\hline & $\begin{array}{c}\text { Fault } \\
\text { coverage }(\%)\end{array}$ & $\begin{array}{c}\text { No. of } \\
\text { transitions }\end{array}$ & $\begin{array}{c}\text { Fault } \\
\text { coverage }(\%)\end{array}$ & $\begin{array}{c}\text { No. of } \\
\text { transitions }\end{array}$ & $\begin{array}{c}\text { Fault } \\
\text { coverage }(\%)\end{array}$ & $\begin{array}{c}\text { No. of } \\
\text { transitions }\end{array}$ \\
\hline alloc_outbound & 83.75 & 106 & 76.64 & 66 & 58.37 & 102 \\
\hline chu172 & 85.29 & 94 & 79.41 & 81 & 79.41 & 91 \\
\hline dff & 68.33 & 78 & 55 & 39 & 53.33 & 58 \\
\hline isend & 62.30 & 141 & 56.92 & 130 & 55.38 & 253 \\
\hline nak-pa & 68 & 184 & 65.33 & 150 & 60 & 167 \\
\hline sbuf-send-ct1 & 71.18 & 112 & 63.84 & 70 & 63.27 & 126 \\
\hline sbuf-send-pkt2 & 76.66 & 192 & 74 & 224 & 72 & 223 \\
\hline ram-read & 66.05 & 159 & 58.71 & 152 & 56.88 & 217 \\
\hline
\end{tabular}




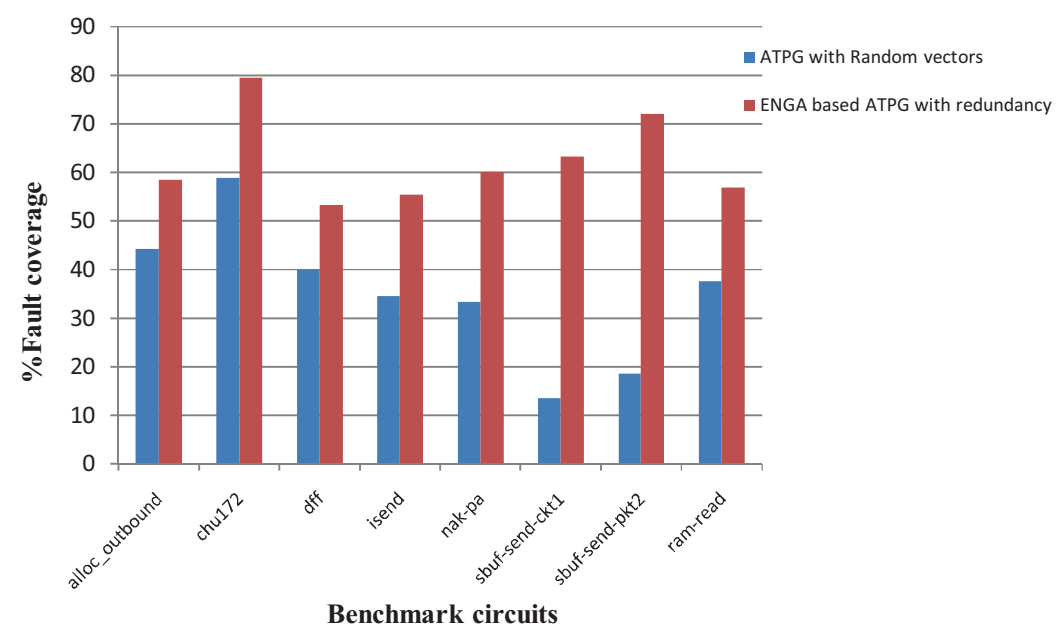

Figure 3. Comparison of fault coverage.

fuzzy delay model based fault simulator. The test validation using fuzzy delay model is more accurate than unit delay model and rise/fall delay model.

Figure 3 gives the comparison chart for fault coverage, for two ATPGs, ATPG with random vectors and ENGA based ATPG with redundancy for the various benchmark circuits. ENGA based ATPG with redundancy gives a better fault coverage for all the benchmark circuits.

\section{Conclusion}

The accuracy of the crosstalk delay fault simulator depends on the delay models used in the simulation. In this paper, an event driven crosstalk delay fault simulator based on fuzzy delay model is used to detect non-confluence of settling states, unstable states and oscillations. The fuzzy delay simulator which can handle uncertainty in gate delays is used to validate the test patterns generated for crosstalk delay faults in asynchronous circuits. To optimize the fault coverage and reduce number of transitions, Elitist Non-Dominated Sorting Genetic Algorithm is used in asynchronous sequential test generation. Experimental results for SIS benchmark circuits are reported. Results demonstrate that tests that cause oscillations and non-confluence of settling states are identified and invalidated by the fuzzy delay model based fault simulator. ENGA based ATPG with redundancy produces higher fault coverage and reduced number of transitions for most of the benchmark circuit compared to random vectors and WSGA based ATPG.

\section{References}

Al-Assadi W K and Kakarla S 2009 Design for test of asynchronous NULL Convention Logic (NCL) Circuits. J. Electron Test 25: 117-126

Aragones X, González J L, Moll F and Rubio A 2002 Noise Generation and Coupling Mechanisms in Deep-Submicron ICs. 2002. IEEE Design and Test of Computers 19(5): 27-35

Banks J, Carson J S and Nelson B L 1996 Discrete-event system simulation. New Delhi: Prentice Hall of India

Benchmark Site 22001 http://www.ac.ups.es./ vlsi/petrify/petrify.Html 
Benchmark Site1 2011 http://www.nangate.com/index.php?option=com_content\&task=view\&id=137\& Itemid $=137$

Bhuvaneswari M C 2001 Development of new algorithms for test generation and simulation of stuck-atfaults in logic circuits. Ph.D Dissertation, Bharathiar University

Bhuvaneswari M C and Sivanandam S N 2000 Event driven simulation of asynchronous sequential circuits using novel fuzzy delay model. Proc. of the National Conference on Recent trends in Advanced Computing 125-129

Deb K 2002 Multi-objective Optimization using Evolutionary Algorithms. John Wiley and Sons Ltd

Dubois D and Prade H 1989 Processing Fuzzy temporal knowledge. IEEE Transactions on system, Man and Cybernetics 19(4): 729-744

Giambiasi N, Smaili M and Frydman C 1994 Discrete event simulation with fuzzy dates. Proc. of the European Simulation Symposium 225-229

Jayabharathi R, d'Abreu M and Abraham J A 1999 FzCRITIC-A Functional timing verifier using a novel fuzzy delay model. Proc. Int. Conference on VLSI Design 232-235

Jayanthy S, Bhuvaneswari M C and Prabhu M 2013 Simulation based ATPG for low power testing of crosstalk delay faults in asynchronous circuits. Int. J. Comput. Appl. Technol. 48(3): 241-252

Kandel A 1986 Fuzzy mathematical techniques with applications. Addison-Wesley

Kishinevsky M, Kondratyev A, Lavagno L, Saldanha A and Taubin A 1998 Partial-scan delay fault testing of asynchronous circuits. IEEE Transactions On Computer-Aided Design Of Integrated Circuits And Systems 17(11): 1184-1199

Michele Favalli 2005 A fuzzy model for path delay fault detection. IEEE Transaction on VLSI Systems 13(8): 943-956

Shi F and Makris Y 2006 Testing Delay Faults in Asynchronous Handshake Circuits. Proc. of ICCAD '06, November 5-9

Sur-Kolay S, Roncken M, Stevens K, Chaudhuri P P and Roy R 2000 Fsimac: A Fault Simulator for Asynchronous Sequential Circuits. Proc. of the 9th Asian Test Symposium 114-119

Takahashi H, Keller K J, Le K T, Saluja K K and Takamatsu Y 2005 A Method for Reducing the Target Fault list of Crosstalk Faults in Synchronous Sequential Circuits. IEEE Transactions on Computer Aided Design of Integrated Circuits and Systems 24(2): 252-263 